published epidemiological studies among more than 30000 hardmetal and cobalt production workers do not provide any evidence for carcinogenicity in humans at exposures in the range from $10-100 \mu \mathrm{g} / \mathrm{m}^{3}$ - between the tolerable concentration based on animal data and the concentration used in the animal experiments. The implications for risk assessment will be discussed also taking into account additional epidemiological data addressing potential inflammatory or fibrogenic effects with impairment of lung function at higher workplace exposures. Copper is another compound for which in Germany a limit value has been proposed by the MAK (maximal workplace concentration) commission based on animal data. Based on a 28 day rat inhalation study with copper(I) oxide which demonstrated inflammatory effects at concentrations starting around $200 \mu \mathrm{g} / \mathrm{m}^{3}$ a MAK value of $10 \mu \mathrm{g} / \mathrm{m}^{3}$ (respirable fraction) has been derived. However, copper has been used at workplaces for centuries at concentrations even in the $\mathrm{mg}$ range without observation of clear health effects. Potential explanations for these discrepancies as well as requirements of additional health data will be presented.

\section{P.1.32 COMPARISONS OF THE RESULTS FROM ERGONOMIC TOOLS BETWEEN RAPID ENTIRE BODY ASSESSMENT AND QUICK EXPOSURE CHECK IN A STEEL FACTORY} ${ }^{1,2}$ Khongrit Pinyowiwat*, ${ }^{1}$ Soontorn Supapong, ${ }^{1}$ Thanapoom Rattananupong. ${ }^{1}$ Faculty of
Medicine, Chulalongkorn University, Bangkok, Thailand; ${ }^{2}$ Occupational and Environmental
Institute of Nopparat Rajathanee Hospital, Bangkok, Thailand

\subsection{6/OEM-2019-EPI.233}

Conducting ergonomic risk assessment is important as it helps employers identify the significant risks in their workplace and will resulting in adequate preventive measures. Simpler observational techniques is one of the types of ergonomic evaluation tools which is commonly used. It is practical, user-friendly and gathering information from both employees and practitioners. This paper presents comparisons between two observational ergonomics tools for determining ergonomics risk factors for work-related musculoskeletal disorders. The Rapid Entire Body Assessment (REBA) and Quick Exposure Check (QEC) were used to assess 296 employees participating in the study from all 12 sections which involved different work tasks in a steel factory. The results are compared using three risk categories (low, moderate, high). Standardised Nordic questionnaires was also used for evaluating the musculoskeletal disorders and effects on working. Data were gathered using questionnaires and evaluations taken at workstation. The findings show the results analyzed using weighted kappa statistic and prevalence of musculoskeletal disorders among workers.

\section{P.1.36 OCCUPATIONAL COHORT STUDIES: SAFEGUARDING A VALUABLE RESOURCE}

${ }^{1}$ Katherine Venables*, ${ }^{2}$ Nicola Fear, 'Lucy Carpenter, ${ }^{3}$ Thomas Keegan, ${ }^{1}$ Claire Brooks, ${ }^{2}$ Gemma Archer. ${ }^{1}$ University Of Oxford, Oxford, UK; ${ }^{2}$ King's College London, London, UK; ${ }^{3}$ Lancaster University, Lancaster, UK

10.1136/OEM-2019-EPI.234
An occupational cohort study is the most robust epidemiological design for studying the effects of workplace hazards and the findings can be extended to the general environment. A cohort may be time-consuming, expensive, and labour-intensive to set up but, once done, it can be extended forward in time, as well as laterally to incorporate new outcome variables, and it can also support nested case-control studies. It is therefore important that the human and material investment is preserved so that these valuable resources can be fully exploited.

In recent years, the bureaucratic burden on researchers in many countries has increased. In the UK, for example, research ethics, data protection, and data access application procedures have become more cumbersome, with an increase in the number of supporting documents required from researchers. Although fast-track procedures exist, epidemiological studies often require the same formal procedures and oversights as more invasive and potentially dangerous physiological and pharmacological studies.

Fortunately, there are now initiatives which support occupational cohort studies. The UK Medical Research Council (MRC), for example, published in 2014 a review and guidance about maximising the value of UK population cohorts and it has also set up a Cohort Strategic Review Group to pre-assess funding applications for new cohorts and for updates to existing cohorts (http://mrc.ukri.org). As another example, OMEGA-NET has been set up to 'create a network to optimize and integrate occupational, industrial, and population cohorts at the European level' (http://omeganetcohorts.eu/).

We propose that a checklist be defined for assessment of research protocols for new cohorts or updates to existing cohorts, in order to assist official committees in their work and streamline the approval process for both researchers and committees. EPICOH would be wellplaced to draft and promulgate such a checklist, working with interested organisations, such as OMEGA-NET and the UK MRC.

\section{P.1.37 A STUDY OF THE INCIDENCE RATE AND RISK FACTORS OF METABOLIC SYNDROME AMONG WORKERS OF DIFFERENT JOB CATEGORIES IN TAIWAN}

Chen-Chang Yang*, Hsin-Chien Wu*. National Yang-Ming University, Taipei city, Taiwan

\subsection{6/OEM-2019-EPI.235}

Background In Taiwan, the prevalence of metabolic syndrome among people over the age of 20 years is as high as $19.7 \%$. With the increase in age, the prevalence of metabolic syndrome is even higher, with the prevalence being more than 30 years among those aged $45-65$ years. Notably, very limited follow-up studies have examined the incidence and risk factors of metabolic syndrome among workers of different job categories in Taiwan.

Methods We conducted a retrospective follow-up study that included 6,284 Taiwanese subjects who had been working in the same job category for 5 years and who received periodic health checkups at a regional hospital from 2006 to 2017 to better understand the incidence rate and risk factors of metabolic syndrome in Taiwan. All participants' demographic data and health examination data were then analyzed. Metabolic syndrome was diagnosed according to the criteria proposd by 
the US National Cholesterol Education Program (NCEP) Third Report of the Expert Panel on Detection, Evaluation, and Treatment of High Blood Cholesterol in Adults (ATP III).

Results After an average follow-up of 6.0 years, the incidence rate of metabolic syndrome among workers who had been working in the same job category for five years was 32.42 per 1000 person-years. Moreover, the largest number of cases with metabolic syndrome appeared in the transportation industry.

Conclusion The results of this study should help the employers of various industries to better plan health education and monitoring programs of their employees to ameliorate the incidence of metabolic syndrome.

\section{P.1.38 EFFECTS OF PARAOXONASE 1 (PON1) GENOTYPE AND NANOMATERIALS EXPOSURE ON HEART RATE VARIABILITY}

Wei-te Wu, Saou-Hsing Liou. National Institute of Environmental Health Sciences, National Health Research Institutes, Miaoli, Taiwan

\subsection{6/OEM-2019-EPI.236}

Background Although paraoxonase 1 (PON1) is a high-density lipoprotein (HDL) associated antioxidant enzyme that has beneficial effects on atherosclerosis and cardiovascular disease, whether PON1 Q192R polymorphism have any effect on cardiac autonomic function is yet to be investigated. Moreover, there is limited data regarding the influence of NPs exposure on HRV parameters in humans. The purpose of the study was to exam whether PON1 genotype or NPs exposure has an association with HRV levels.

Methods This cross-sectional study recruited 235 workers exposed to NM and 185 non-exposed controls from $14 \mathrm{NM}$ handling plants in Taiwan from 2009 to 2011. For each participant, we collected blood specimens to determine the genotype of the PON1 Q192R polymorphism and PON1 paraoxonase and arylesterase activities. In addition, short-term HRV was tested.

Results The results showed that PON1 Q192R genotype and PON1 paraoxonase/arylesterase activities associated with HRV, and was particularly noteworthy in RMSSD and HF. The relationship between NPs exposure and HRV was only found in workers exposed to nano-Ag, but the apparent relationship between NPs exposure and HRV is lacking.

Conclusions It is a novel finding that both RR genotype of PON1 (Q192R) and activities of PON1 increased with HRV levels. PON1 Q192R genotype may play an important role in cardiac protection. Considering the global prevalence of NMs market, further studies of NPs-induced cardiac events and stresses still are required to establish.

\section{P.1.40 FARMER'S SUICIDE WITH THE USE OF PESTICIDES IN DIFFERENT AREAS IN SPAIN}

${ }^{1}$ Guanlan Zhao, ${ }^{1}$ Elena Ronda, ${ }^{2}$ Gregorio Barrio, ${ }^{3}$ Enrique Regidor. ${ }^{1}$ University of Alicante, Alicante, Spain; ${ }^{2}$ National School of Public Health, Instituto de Salud Carlos III, Madrid, Spain; ${ }^{3}$ Complutense University of Madrid, Madrid, Spain

\subsection{6/OEM-2019-EPI.237}

Introduction There is considerable interest in the potential relation of suicide with the pesticide. Agriculture is the leading professional sector for pesticide use in the world with a turnover of about 40 billion US dollars in 2010. According to the WHO report in 2014, autointoxication with agricultural pesticides contributes greatly to the global burden of suicide. The aim of this study is to evaluate whether suicide of farmers was associated with the use of pesticide in different areas during the period 2001-2011 in Spain.

Methods The cohort study followed 9.5 million men in Spain from 2001 to 2011. Participants aged 20-64 years were employed in 2001. The study subjects were grouped as farmers and non-farmers. We divided Spain into two areas depending on both geographical location and the mean annual amount of pesticides used: southeast Spain and the rest of Spain. The southeast Spain shows higher use of pesticides than the rest of Spain. For three major causes of death (suicide, unintentional accidents and rest of causes of death), we estimated the age-standardized mortality rates per 1000,000 person-years of follow-up, the ratio of age-standardized mortality rates with $95 \%$ confidence intervals (CI) between farmers and non-farmers in two areas were calculated.

Results With respect to the rest area of Spain, farmers in the southeast showed a lower mortality rate of suicide and unintentional accidents, which mortality rate ratios were 0.74 (95\% CI $0.65-0.85)$ and 0.57 (95\% CI $0.52-$ $0.62)$.

Conclusion Our findings do no support a possible association between pesticide use and suicide of farmers in Spain.

\section{P.2.01 EPIDEMIOLOGICAL STUDIES OF THE PORTON DOWN VETERANS: AN UPDATE OF CANCER INCIDENCE AND MORTALITY TO 2018}

${ }^{1}$ Gemma Archer, ${ }^{2}$ Thomas Keegan, ${ }^{3}$ Claire Brooks, ${ }^{3}$ Lucy Carpenter, ${ }^{3}$ Katherine Venables, ${ }^{1}$ Nicola Fear. ${ }^{1}$ King's College London, London, UK; ${ }^{2}$ Lancaster University, UK; ${ }^{3}$ Oxford University, UK

\subsection{6/OEM-2019-EPI.238}

Introduction An update to the Porton Down Veterans Cohort study is planned for 2018-2021.

Background Chemical warfare agents continue to be deployed by rogue states and terrorists, e.g. in Syria, Iraq, Tokyo, and Salisbury UK, yet their impact on long-term health is largely unknown. The Porton Down cohort comprises of 18276 men who took part in the 'human volunteer programme' of chemical warfare agent research at Porton Down, UK, between 1941 to 1989 , and a comparison group of 17600 men who also served in the military. An original study linked veterans' records to national registry data on cancer incidence and mortality up to 2004. By the end of 2004, 7306 of the Porton Down veterans and 6900 of the comparison cohort had died. The results showed Porton Down veterans had no overall excess of cancers (rate ratio $(\mathrm{RR})=1.00$, 95\% CI 0.95-1.05) and a small (6\%) excess of all-cause mortality $(\mathrm{RR}=1.06$, 1.03-1.10) when compared with non-Porton Down veterans. Porton Down veterans had higher rates of certain cancers (e.g. ill-defined malignant neoplasms, and in situ neoplasms) and specific causes of death (e.g. genitourinary, circulatory and external causes).

Objectives The updated study aims to replicate the original analyses but with an additional 15 years of follow-up - estimated to increase the number of deaths available for study to c 22 200. This will improve statistical power, allowing 